\title{
Current assessment of the (dys)function of macrophages in endometriosis and its associated pain
}

\author{
Sarah Brunty, Nalini Santanam \\ Department of Biomedical Sciences, Joan C. Edwards School of Medicine, Marshall University, Huntington, WV, USA \\ Correspondence to: Nalini Santanam, PhD, MPH. Professor, Department of Biomedical Sciences, Joan C. Edwards School of Medicine, Marshall \\ University, 1700 3rd Ave, Huntington, WV 25755, USA. Email: santanam@marshall.edu. \\ Provenance: This is an invited article commissioned by the Section Editor Dr. Hengwei Liu (Department of Obstetrics and Gynecology, Zhongnan \\ Hospital of Wuhan University, Wuhan, China). \\ Comment on: Forster R, Sarginson A, Velichkova A, et al. Macrophage-derived insulin-like growth factor-1 is a key neurotrophic and nerve-sensitizing \\ factor in pain associated with endometriosis. FASEB J 2019;33:11210-22.
}

Submitted Dec 05, 2019. Accepted for publication Dec 19, 2019.

doi: 10.21037/atm.2019.12.119

View this article at: http://dx.doi.org/10.21037/atm.2019.12.119

Endometriosis is a gynecological disease that affects 1 in 10 women worldwide and is characterized by the presence of lesions made up of endometrial cells (ECs) outside of the uterus. Symptoms associated with endometriosis include pain and infertility. It was realized in early last century that endometriosis is an inflammatory disease. Search for the mediators of this inflammation had been one of the key areas of research in understanding the etiology of this disease. Pioneering work by Halme, Olive, and Arici's groups provided evidences for an inflammatory peritoneal milieu and the role for macrophages in endometriosis (1-3). Publications as early as in the 1980 s showed that the peritoneal macrophages are present at higher levels in women with endometriosis-associated infertility when compared to infertile women due to causes other than endometriosis or normal women (4). Just a few years later, Badawy et al. also examined the peritoneal fluid (PF) of women with endometriosis and those who were infertile and showed that macrophages and lymphocytes were the dominant cells in their fluid. They also noted that prostaglandins, $\mathrm{PGF}_{2 \alpha}$, and $\mathrm{PGE}_{2}$ were also increased in the fluid (5).

In general terms, macrophages are white blood cells in the immune system that digests cellular debris, foreign substances, cancer cells, etc. Their recruitment into the peritoneal cavity (PC) in women with endometriosis might be in response to the retrograde menstruation and presence of the apoptotic ECs. However, macrophages are also known to be pro-inflammatory, which is one of the reasons why investigators were curious to understand the role of these immune cells in the pathogenesis of endometriosis as well as its associated symptoms, pain and infertility. Interestingly, the clue to this came from studies in the cardiovascular field. In 1994, researchers from Japan sought to determine what triggers the macrophage activation in endometriosis patients, as well as what immunologic conditions occur in their PC. They measured the amount of phospholipase A2, cholesterol fractions [high-density lipoprotein (HDL), low-density lipoprotein (LDL), and very low-density lipoprotein (VLDL)], interleukins (IL)1 and 6 , granulocyte-macrophage-colony stimulating factor (GM-CSF), and phosphatidylserine in the PF. When comparing control PF and endo PF, they found no changes in phospholipase A2 but did see an increase in IL-6. They concluded that HDL or LDL may play a role in macrophage activation in women with endometriosis and that the increased IL- 6 observed suggests immunological events causing infertility in these women (6).

At the same time, our group consisting of cardiovascular researchers, utilized the research that had already been done in atherosclerosis, where oxidized LDLs are involved in macrophage activation and formation of foam cells, a key event in atherosclerosis (7-9), used this knowledge and examined similar mechanisms in endometriosis $(10,11)$. We found similarities between components present in the $\mathrm{PF}$ of women with endometriosis and those present in the atherosclerotic artery, for example increased presence of cytokines, chemokines, and growth factors (12). It is known 
that through their scavenger receptor(s), SR-A or CD36, macrophages can play an important role in the uptake and clearance of modified proteins (including oxidized LDL) which may be formed due to the inflammatory processes (13). Oxidized LDL is antigenic, proinflammatory, alters many cell types, and also induces and releases IL-1 from macrophages (14). These findings prompted us to look for the presence of oxidatively modified lipid-protein complexes in the $\mathrm{PF}$, which might be ligands for macrophage scavenger receptor(s). We found that in the PF of women with endometriosis, there was an increase in lysophosphatidylcholine (a known monocyte and T-lymphocyte chemotactic agent) and oxidized LDL, suggesting an oxidative milieu in the PC of women with endometriosis. LDL isolated from endometriotic PF was also more readily oxidized than those isolated from control PF or plasma of patients with and without endometriosis. Presence of activated macrophages and oxidative stress in the PC resulted in lower levels of the antioxidant, vitamin $\mathrm{E}$ in the endo $\mathrm{PF}$ than in control PF. These results showed strong evidence for the occurrence of oxidative stress in the PF of women with endometriosis and that the oxidized lipoproteins present in the PF possess properties that suggest their involvement in the pathogenesis of endometriosis (12). It was also noted that monocyte chemotactic protein-1 (MCP-1), a potent monocyte/ macrophage chemoattractant and activator, was present at higher levels in the PC of women with endometriosis. Interestingly, mesothelial cells, which are one of the key cells present in the $\mathrm{PC}$ and major producers of chemokines, produced more MCP-1 and IL-6 compared to endometrial epithelial and stromal cells. Co-culturing of the mesothelial cells with endometrial epithelial cells increased the production of MCP-1, IL-6 and IL-8 in the PC (15). Interestingly $M C P-1$ was also induced by oxidized LDL and $\mathrm{PF}$ from women with endometriosis (16).

With the knowledge that the PF is rich in inflammatory mediators and pain-inducing prostaglandins and lipid peroxides, and the endometriotic tissue is innervated with nociceptors, we hypothesized that oxidatively-modified lipoproteins present in the PF are the major source of nociceptive molecules involved in pain associated with endometriosis. We found that there was an increased presence of enzymatically derived prostaglandins and oxidized lipids or non-enzymatically and free-radical derived 8 -isoprostanes in the PF of women with endometriosis when compared to control PF. We also found that $90 \%$ of endo $\mathrm{PF}$, in contrast to $45 \%$ of control PF were positive for the presence of lipoproteins (LDL or HDL). Using a rodent model and various pain behavior assays (changes in body temperature, Hargreaves paw withdrawal assay), we found that similar to PF from women with endometriosis, oxidatively modified LDL injected into the paws of rodents showed a nociceptive response. Preparations of these oxidatively modified LDLs in the presence of antioxidants or NSAIDs prevented the nociceptive response. These LDL preparations similar to PF from women with endometriosis also increased the levels of inflammatory genes such as $I L-6$ and pain regulating genes such as NGF, PTGES3, MCP-1, voltage-gated sodium channels. Incidentally, these inflammatory and nociceptive genes are also upregulated by cyclooxygenase derived prostanoids during inflammation. Overall, these findings supported the presence of the oxidized-lipid molecules in the PF of women with endometriosis and their potential role in pain associated with endometriosis $(17,18)$. All these studies re-affirmed that the oxidative milieu in the PC of women with endometriosis can recruit macrophage and induce inflammation.

When comparing the immune cells within the PC, it was observed that control women had lesser PF and very few monocyte-like cells. In contrast, there was more PF and more numbers of monocyte-macrophages in the PF of women with endometriosis. It should be noted that macrophages gain scavenger function when adherent (13) and hence it was observed that when PF macrophages from endometriosis patients were made adherent (by plating on tissue culture plates) they were able to take up and degrade oxidatively modified lipoproteins more efficiently than similar macrophages from control women $(19,20)$. This observation suggests that despite the increased inflammatory milieu, the peritoneal macrophages are unable to scavenge due to their non-adherent nature. This lack of scavenger function might be related to the inability of the monocyte/macrophages to adhere to the mesothelial layer of the PC or the inability of the mesothelium to be conducive for macrophage adhesion (Figure 1). This needs to be further investigated. However, it should be noted, that though these macrophages are non-adherent, they still continue to release inflammatory, oxidative and nociceptive mediators and play a role in endometriotic lesion growth as well as in nociception.

In the recently published study, Forster et al. (21) has added to this knowledge of the role of macrophages in endometriosis and pain. When a mouse model for endometriosis was treated with liposomal clodronate 


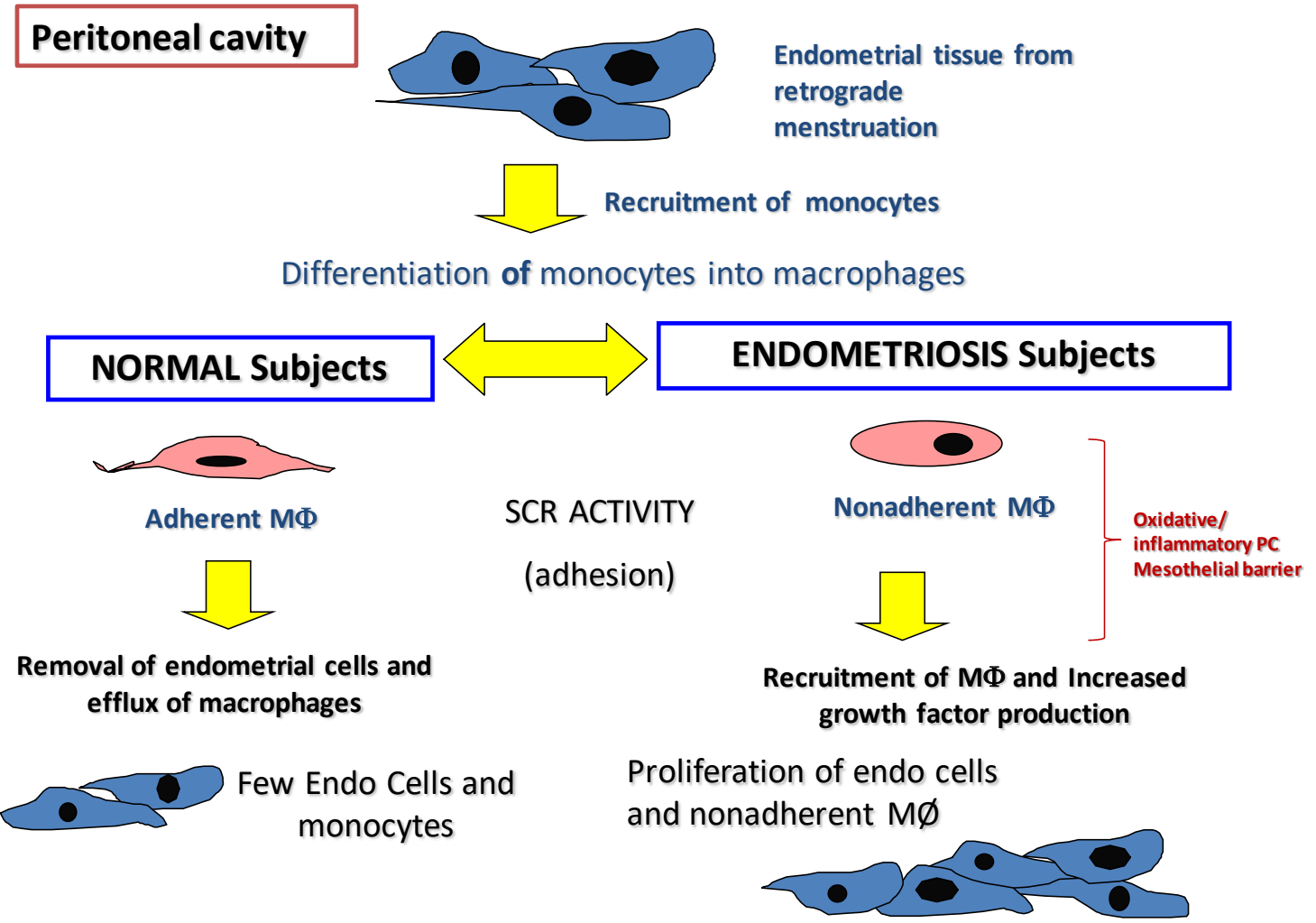

Figure 1 Schematic representation of the macrophage (dys)function in endometriosis. SCR, scavenger receptor; PC, peritoneal cavity.

(macrophage depletion) there was a significant depletion of large peritoneal macrophages (LpMs) and transient monocyte-derived macrophages (MDMs) as well as an increase in the number of monocytes. Assessment of pain behavior in the treated $v s$. non-treated mice showed that in the mice with endometriosis (non-treated mice), had an increase in spontaneous grooming, decreased activity and abdominal retraction, and decreased pain withdrawal thresholds. Once these mice were treated with liposomal clodronate, the levels of grooming declined and reduced the paw withdrawal threshold, but the level of activity was unchanged. They concluded that it appeared that macrophages and macrophage-derived mediators activate these changes in sensory behavior.

In accordance with their previous study showing an induction of Cox 2 in the endometrial lesions, spinal cords and brain (22), they observed in the current study that mice with endometriosis had an increased mRNA expression of Cox-2 as well as $T n f-\alpha$ and that these levels were reduced following macrophage depletion. In order to identify macrophage-derived factors involved in pain, the authors, modelled, endometriosis-associated macrophages (EAMs), using PF from women with endometriosis and pain, and found that the mRNA expression of $B D N F$ and $I G F-1$ was elevated in the EAMs. An increase in IGF-1 correlated with the levels of pain in these women. Macrophage-derived IGF-1 also enhanced the sprouting of the dorsal root ganglion (DRG) neurons thus increasing nociception. It was concluded that the neurotropic effects seen in the PF and macrophages from women with endometriosis is partially mediated by IGF-1 and by inhibiting IGF-1 in mice using linsitinib, pain behavior such as abdominal retraction and paw withdrawal threshold were higher compared to vehicle treated control mice.

Overall, Forster et al. (21) have shown a major role for peritoneal macrophages in endometriosis-associated hyperalgesia. Their studies further identified that IGF1 is the key macrophage-derived mediator responsible for hyperalgesia, since inhibition of IGF-1 was able to reduce nerve growth as well as lower the expression of nociceptive genes in vitro. Hence, these authors concluded that macrophages or factors derived from activated macrophages 
may be good targets for therapy to reduce pain in women with endometriosis.

The question remains of how these new findings by Forster et al. fits in and adds to the knowledge that is already known in the field of endometriosis research. Some of the factors identified by our team in the PC (oxidized lipids, inflammatory mediators) can all be macrophagederived factors that lead to the findings observed by Forster et al. Our placebo controlled clinical trial of supplementing women with pelvic pain and endometriosis, vitamin $\mathrm{E}$ $(1,200 \mathrm{IU})$ and vitamin $\mathrm{C}(1,000 \mathrm{mg})$ combination or just placebo daily for 8 weeks before surgery, showed that antioxidant supplementation, improved everyday pain in $43 \%$ of patients compared to the placebo group. Dysmenorrhea and dyspareunia were also decreased by $37 \%$ and $24 \%$ in patients, respectively. There was also a significant decrease in PF inflammatory molecules, such as MCP-1 and IL-6 after antioxidant therapy (23). It would be interesting to explore whether a dual combination therapy of depleting macrophages or inhibiting IGF-1 by linsitinib, along with vitamin $\mathrm{E} / \mathrm{C}$ would provide more attenuation of the pain that women with endometriosis experience, compared to each of these treatments alone. Also, it would be interesting to investigate if this combination therapy would also decrease the lesions in these women and provide a more tailored treatment for endometriosis. These questions, and potential therapies, are only the tip of the iceberg in questions and things to explore in endometriosis, as many things are yet to be discovered in this elusive disease.

\section{Acknowledgments}

The authors thank Dr. Brenda Mitchell, MD and Dr. David Jude, MD; Department of Obstetrics and Gynecology, Joan C. Edwards School of Medicine for their support of our endometriosis research. Sarah Brunty acknowledges PhRMA Grant 218218 Pre-Doctoral Fellowship.

\section{Footnote}

Conflicts of Interest: The authors have no conflicts of interest to declare.

Ethical Statement: The authors are accountable for all aspects of the work in ensuring that questions related to the accuracy or integrity of any part of the work are appropriately investigated and resolved.

\section{References}

1. Oral E, Olive DL, Arici A. The peritoneal environment in endometriosis. Hum Reprod Update 1996;2:385-98.

2. Olive DL, Weinberg JB, Haney AF. Peritoneal macrophages and infertility: the association between cell number and pelvic pathology. Fertil Steril 1985;44:772-7.

3. Halme J, White C, Kauma S, et al. Peritoneal macrophages from patients with endometriosis release growth factor activity in vitro. J Clin Endocrinol Metab 1988;66:1044-9.

4. Haney AF, Muscato JJ, Weinberg JB. Peritoneal fluid cell populations in infertility patients. Fertil Steril 1981;35:696-8.

5. Badawy SZ, Cuenca V, Marshall L, et al. Cellular components in peritoneal fluid in infertile patients with and without endometriosis. Fertil Steril 1984;42:704-8.

6. Ueki M, Tsurunaga T, Ushiroyama T, et al. Macrophage activation factors and cytokines in peritoneal fluid from patients with endometriosis. Asia Oceania J Obstet Gynaecol 1994;20:427-31.

7. Quinn MT, Parthasarathy S, Steinberg D. Endothelial cell-derived chemotactic activity for mouse peritoneal macrophages and the effects of modified forms of low density lipoprotein. Proc Natl Acad Sci U S A 1985;82:5949-53.

8. Hogg N, Struck A, Goss SP, et al. Inhibition of macrophage-dependent low density lipoprotein oxidation by nitric-oxide donors. J Lipid Res 1995;36:1756-62.

9. Parthasarathy S, Santanam N. Mechanisms of oxidation, antioxidants, and atherosclerosis. Curr Opin Lipidol 1994;5:371-5.

10. Santanam N, Murphy AA, Parthasarathy S. Macrophages, oxidation, and endometriosis. Ann N Y Acad Sci. 2002;955:183-98; discussion 19-200, 396-406.

11. Santanam N, Song M, Rong R, et al. Atherosclerosis, oxidation and endometriosis. Free Radic Res 2002;36:1315-21.

12. Murphy AA, Santanam N, Morales AJ, et al. Lysophosphatidyl choline, a chemotactic factor for monocytes/T-lymphocytes is elevated in endometriosis. J Clin Endocrinol Metab 1998;83:2110-3.

13. Kim JG, Keshava C, Murphy AA, et al. Fresh mouse peritoneal macrophages have low scavenger receptor activity. J Lipid Res 1997;38:2207-15.

14. Steinberg D, Parthasarathy S, Carew TE, et al. Beyond 
cholesterol. Modifications of low-density lipoprotein that increase its atherogenicity. $\mathrm{N}$ Engl J Med 1989;320:915-24.

15. Song M, Karabina SA, Kavtaradze N, et al. Presence of endometrial epithelial cells in the peritoneal cavity and the mesothelial inflammatory response. Fertil Steril 2003;79 Suppl 1:789-94.

16. Rong R, Ramachandran S, Santanam N, et al. Induction of monocyte chemotactic protein-1 in peritoneal mesothelial and endometrial cells by oxidized low-density lipoprotein and peritoneal fluid from women with endometriosis. Fertil Steril 2002;78:843-8.

17. Ray K, Fahrmann J, Mitchell B, et al. Oxidation-sensitive nociception involved in endometriosis-associated pain. Pain 2015;156:528-39.

18. Wright KR, Mitchell B, Santanam N. Redox regulation of microRNAs in endometriosis-associated pain. Redox Biol 2017;12:956-66.

Cite this article as: Brunty S, Santanam N. Current assessment of the (dys)function of macrophages in endometriosis and its associated pain. Ann Transl Med 2019;7(Suppl 8):S381. doi: 10.21037/atm.2019.12.119
19. Murphy AA, Palinski W, Rankin S, et al. Macrophage scavenger receptor(s) and oxidatively modified proteins in endometriosis. Fertil Steril 1998;69:1085-91.

20. Murphy AA, Palinski W, Rankin S, et al. Evidence for oxidatively modified lipid-protein complexes in endometrium and endometriosis. Fertil Steril 1998;69:1092-4.

21. Forster R, Sarginson A, Velichkova A, et al. Macrophagederived insulin-like growth factor-1 is a key neurotrophic and nerve-sensitizing factor in pain associated with endometriosis. FASEB J 2019;33:11210-22.

22. Greaves E, Horne AW, Jerina H, et al. EP2 receptor antagonism reduces peripheral and central hyperalgesia in a preclinical mouse model of endometriosis. Sci Rep 2017;7:44169.

23. Santanam N, Kavtaradze N, Murphy A, et al. Antioxidant supplementation reduces endometriosis-related pelvic pain in humans. Transl Res 2013;161:189-95. 